\title{
TRATAMENTO HOMEOPÁTICO E SUA IMPLANTAÇÃO NO SUS
}

\author{
Aline de Oliveira Galvão da Silva ${ }^{1}$ \\ Leonardo Guimarães de Andrade ${ }^{2}$ \\ Michel Santos da Silva ${ }^{3}$ \\ Fabiana Sousa Pugliese ${ }^{4}$
}

RESUMO: Em todo o mundo, os usuários de serviços médicos estão cada vez mais demonstrando Interesse em Medicina Tradicional e Complementar (MTC). "Homeopatia é Incluído tradicionalmente na medicina Tradicional e Complementar, caracterizado por sua resolução, Baixo custo, ampla cobertura e inegável reconhecimento social. Em maio de 2006, publicado na "Política Nacional de Abordagem Integrada e (PNPIC), que também inclui a homeopatia no sistema médico unificado Saúde (SUS). "A Homeopatia é uma ciência baseada na capacidade do organismo em manter o equilíbrio, graças à força vital que regula nossas funções e reações automaticamente, com objetivo de devolver a saúde" (SILVA, 2020, P. 3). "Samuel Hahnemann foi o principal responsável por esse ramo da medicina e acreditava que o que causa o mal (medicamento capaz de produzir determinados sintomas no indivíduo sadio) seria capaz de curar o mal (doença) " (SILVA, 2020, P. 3). Com base em sua experiência, três princípios universais foram estabelecidos: (I) Lei da similaridade, onde o semelhante cura o semelhante; (2) experimentos em homens saudáveis; e (3) doses pequenas, usando o medicamento diluído. Ele percebeu que quando ele diluiu a dose, o efeito $\mathrm{O}$ curativo persiste e o agravamento tende a diminuir e desaparecer (SILVA, 2020). Com a inclusão da homeopatia Serviço público nas últimas décadas por meio da de gestores, o primeiro Fórum Nacional Homeopático em 2004 foi destinado a promover uma ampla gama de debates com diferentes departamentos, Ações diretas ou indiretas foram formuladas, Assistência homeopática, ensino e pesquisa no SUS, fazendo assim, com que as pessoas tenham um conhecimento melhor sobre a homeopatia em medicamentos pela rede pública. Desde então, "start" é acionado, e Homeopatia lançou as bases no SUS, no entanto, existem muitas dificuldades e constatase que essa prática se tornou norma (souza, leite, yoshida, santos, 2019).

Palavras- chaves: Homeopatia. SUS. Medicamento.

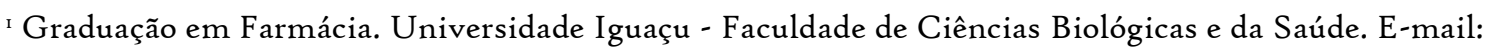
alinegalvao.18@hotmail.com

${ }^{2}$ Co-autor.

3 Orientador.

${ }_{4}^{4}$ Orientadora.
} 


\section{INTRODUÇÃO}

Existem muitos motivos pelos quais os pacientes procuram a homeopatia, incluindo os seguintes motivos: (I) Outros tratamentos não alcançaram os resultados esperados; (2) A visão de ter menos efeitos adversos em tratamentos mais "naturais"; (3) Homeopatas fornecem cuidados e atenção. A fim de melhorar a eficácia do tratamento da homeopatia, é necessário que os pacientes compreendam totalmente a particularidade dos medicamentos prescritos para eles. Nesse caso, o cuidado com a medicação desempenha um papel importante para garantir que os pacientes sejam bem monitorados durante o tratamento.

“Lei no 8.08o de 19 de setembro de 1990 define o SUS (Sistema Único de Saúde) Saúde) é um conjunto de ações e serviços de saúde geridos por instituições públicas municipais, Estadual e federal" (SOUZA, LEITE, YOSHIDA, SANTOS, 2019, PI). De acordo com as diretrizes do artigo 198 da Constituição Federal onde diz que os serviços de saúde devem ser de acesso universal para todos e a nível de assistência, oferecendo serviços de saúde curativos e preventivos, de forma individuais e coletivos se necessário, sem discriminação da população. No SUS a medicina tradicional e a medicina complementar são usadas. Essas práticas envolvem principalmente homeopatia, acupuntura, Fitoterapia e medicina humana. A medicina tradicional e complementar é fornecida pelo sistema público de saúde desde a década de 1980, mas se ampliou e intensificou com a Aprovação da PNPIC (Política Nacional de Práticas Integradas e Complementares) que assegura o acesso aos usuários do SUS à medicina tradicional chinesa/acupuntura, homeopatia, fitoterapia e termalismo social (SOUZA, LEITE, YOSHIDA, SANTOS, 2019).

Com a inclusão da homeopatia Serviço público nas últimas décadas por meio da de gestores, o primeiro Fórum Nacional Homeopático em 2004 foi destinado a promover uma ampla gama de debates com diferentes departamentos, Ações diretas ou indiretas foram formuladas, Assistência homeopática, ensino e pesquisa no SUS, fazendo assim, com que as pessoas tenham um conhecimento melhor sobre a homeopatia em medicamentos pela rede pública. Desde então, "start" é acionado, e Homeopatia lançou as bases no SUS, no entanto, existem muitas dificuldades e constata-se que essa prática se tornou norma (SOUZA, LEITE, YOSHIDA, SANTOS, 2019). 
A OMS incentiva o desenvolvimento de programas de homeopatia, com o objetivo de aumentar sua disponibilidade no sistema público de saúde global com o auxílio de terapias clássicas, pois a homeopatia é considerada uma alternativa eficaz e segura para o tratamento de doenças crônicas. Com o objetivo de estimular e apoiar projetos de homeopatia, ensino e pesquisa e outras práticas não convencionais em diversos campos do SUS, o Ministério da Saúde aprovou em 2006 a "Política Nacional de Práticas Unificadas e Complementares no Sistema Único de Saúde"

"Nas faculdades de Farmácia do Brasil, o ensino de farmacotécnica homeopática passou a ser obrigatório a partir de 1952, com a Lei 1.552. De acordo com um levantamento de dados realizado em 200I, metade dos cursos de Farmácia no Brasil oferecia a disciplina de farmacotécnica homeopática em caráter optativo.” (NECKEL et al., 2010).

“Atualmente, com a reformulação do ensino em praticamente todas as faculdades de Farmácia, por efeito das novas diretrizes curriculares elaboradas pelo Conselho Nacional de Educação, o ensino de homeopatia voltou a ser o foco das atenções." (NECKEL et al., 2010).

O tratamento medicamentoso está intimamente relacionado ao papel dos farmacêuticos na prática homeopática. Ele deve exercer seu papel de auxiliar e promover o uso Racional de medicamentos e supervisionar o tratamento de perto, estabelecendo assim uma uma "conexão" com o paciente. Medicamento Homeopáticos, por ser considerados como uma terapia "não convencional", então deve haver atendimento claro e expandido para pacientes usando esse método de tratamento.

Ainda segundo (MESSIAS MCF, 2015), a intervenção do farmacêutico faz-se fator primordial na prevenção do uso irracional de medicamentos e para que tal prática não ganhe forças é necessário um acompanhamento farmacoterapêutico individualizado do paciente, garantindo a segurança e efetividade no uso do medicamento. 


\section{HOMEOPATIA}

"Nos dias atuais, intensifica-se os estudos que identificam a homeopatia como método eficiente contra uma série de doenças crônicas, e como instrumento a ser utilizado para profilaxia e cura de certas epidemias." (LIRA et al., 2020, pI).

Nesse contexto, a homeopatia foi fundada por Samuel Hahnemenn, nasceu na cidade de Meissen, Alemanha, no dia ro de abril de 1755 (1755-1843) um médico que no século XVIII, mais especificamente no ano de 1779, funda o método terapêutico que hoje é conhecido como homeopatia. Porém, mesmo esse método sendo aprovado pela OMS, ainda assim são frequentes as controvérsias perante a sociedade atual sobre a eficácia na cura de doenças crônicas, sendo que um dos principais fundamentos da homeopatia, é a lei dos semelhantes, sendo essa caracterizada pela utilização das mesmas substâncias que provocam determinadas enfermidades, para o progresso de uma cura (LIRA et al., 2020).

IMAGEM r: Samuel Hahnemenn - Fundador da Homeopatia.

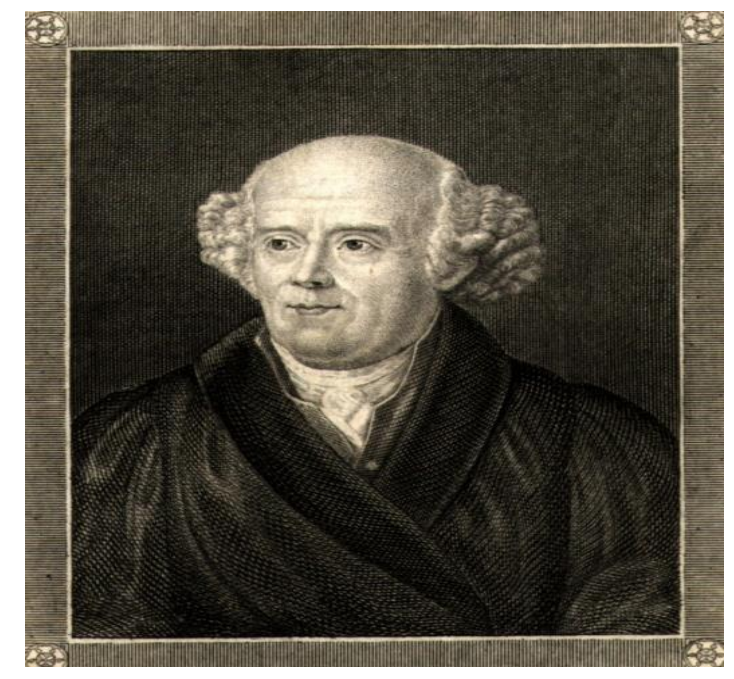

FONTE:http://t3.gstatic.com/licensedimage?q=tbn:ANd9 $\mathrm{GcQS}_{74} \mathrm{FZIouCMdT}_{7} \mathrm{~N}_{3} \mathrm{H}_{2}$ w6 6 BtKYHtwrglGF ${ }_{2 g K e u}{ }_{4 R}$ Rn537Cy7dPjke332u6Wqq.

"A homeopatia utiliza drogas de origem vegetal, mineral e animal. O medicamento age por estímulo ao processo natural de cura do organismo, é seguro quando supervisionado 
pela equipe de saúde, mas apresenta efeitos adversos como qualquer outro medicamento" (SILVA, 2020, $\left.\mathrm{P}_{7}\right)$.

Validade desde o século XX, pelo Conselho Federal de Medicina em 1980, a homeopatia ganha simpatizantes cada vez mais, pois mostra relevância e fundamentos na continuidade de pesquisas científicas, que provam a eficiência dessa forma terapêutica alternativa. Diante de tais fatos é imprescindível afirmar que a homeopatia é um tratamento eficaz e terapêutico, ou seja, a homeopatia encontra-se atrelada a promoção da homeostase do indivíduo, garantindo com que este não adoeça (LIRA et al., 2020).

"No Brasil, esta terapêutica foi trazida no ano de 1840 , por Bento Mure, sendo reconhecida como especialidade médica, pelo Conselho Federal de Medicina, em i98o e especialidade farmacêutica, pelo Conselho Federal de Farmácia, em 1992.” (SANTOS, SÁ, 2014, Ip).

$\mathrm{Na}$ medicina homeopática o homem é visto como sujeito da sua saúde e as consultas são definidas pelo progresso do autoreconhecimento do paciente, fazendo com que assim, a libertação da autonomia pelo paciente em relação ao próprio corpo. E também preserva a ideia de que o medicamento deve ser único e se ajustar a necessidade de cada indivíduo, isso faz com que mesmo que seja a mesma patologia, dois pacientes recebam medicamentos diferentes (SANTOS, SÁ, 2014).

Basicamente a homeopatia é um método donde o profissional habilitado a prescrição de medicamentos, e que esse profissional também tenha formação em medicamentos homeopáticos, prescreve ao paciente doses reduzidas de algum composto, o composto é determinado pela doença. Em outros casos que a dose é elevada, iria promover sintomas análogos da enfermidade que queira combater.

A anamnese homeopática considera muitos fatores do indivíduo e de forma integral, avaliando todas as queixas, alterações fisiológicas, sinais, histórico familiar, hábitos e sintomas mentais. Conhecer os hábitos do paciente também é importante nessa anamnese. $\mathrm{Na}$ homeopatia a singularidade é essencial e fundamental e é isso que define a terapêutica aplicada e qual será o medicamento mais efetivo. 
“Tais medicamentos empregam substâncias dos reinos animal, vegetal e mineral, sob as recomendações da Farmacopeia Homeopática Brasileira (FHB).” (PNPIC, 2015).

Medicamento homeopático é toda apresentação farmacêutica destinada a ser ministrada segundo o princípio da similitude, com intuito final de prevenção e terapêutico, que é obtido pelo método de diluições seguidas de agitações e/ou triturações sucessivas. $\mathrm{O}$ medicamento homeopático pode ser manipulado sob forma líquida, glóbulos, pó e em tabletes.

De forma geral, os remédios homeopáticos são feitos por meio da dinamização. Ou seja, as soluções diluídas são agitadas para libertar a energia que deve ser usada no tratamento. Isso é feito por meio de choques fortes e ritmados.

\section{I.I HOMEOPATIA NO SUS}

No ano de 1988 o Sistema Único de Saúde, conhecido em todo o mundo como SUS, por ocasião da promulgação da Constituição da República Federativa do Brasil, foi fundado no Brasil. O SUS oferece desde então, a todo cidadão acesso de forma integral e gratuitamente serviços de saúde. Apesar de todo conflito político, o SUS é considerado um dos melhores sistemas de saúde pública, esse reconhecimento é universal. Esse sistema oferece benefícios em média I8o milhões de cidadãos brasileiros e concretiza em média 2,8 bilhões de atendimentos anualmente. São realizados tanto procedimentos simples, como procedimentos complexos, como por exemplo, transplantes de órgãos.

Logo após a criação do SUS, alguns estados no Brasil iniciaram a prestação de serviços homeopáticos aos cidadãos que utilizam o SUS. A portaria 971/20o6 foi editada e assegura que o SUS fornece à população acesso a medicina complementar, ou seja, homeopatia esta inclusa nessa lista, assim como acupuntura, fitoterapia e Termalismo Social/Crenoterapia.

“A entrada da Homeopatia, no SUS, é resultado da Política Nacional de Práticas Integrativas e Complementares (PNPIC), criada pela Portaria 971/2006, do Ministério da Saúde.” (GUTIERREZ, 2008). 
No ano de 2007, em dezembro foi publicada a portaria 3.237, aonde o Ministério da Saúde engloba os medicamentos homeopáticos da Farmacoterapia Homeopática Brasileira (em média 450 medicamentos) para serem oferecidos aos pacientes do SUS, em concessão com oque recomenda a PNPIC, proposto por Hahnemann no Organon. A PNPIC tem o intuito de permitir o acesso aos serviços e produtos das Práticas Integrativas e Complementares (PICs), de forma segura, eficaz e com atuação multiprofissional, em conformidade com os princípios e diretrizes do SUS.

“A OMS tem incentivado o desenvolvimento de projetos homeopáticos que visem incrementar sua disponibilidade junto aos sistemas públicos de saúde mundiais, de forma coadjuvante aos tratamentos clássicos, por ser a homeopatia considerada uma alternativa eficiente e segura ao tratamento das doenças crônicas." (NECKEL et al., 2010).

A homeopatia pode ser combinada com tratamento de medicina tradicional e isso é até recomendado pela Organização Mundial de Saúde no tratamento de doenças orgânicas graves, como câncer, malária, tuberculose, entre outras.

\subsection{CUSTO-BENEFÍCIO DO TRATAMENTO HOMEOPATA}

O custo-benefício da homeopatia é acessível a todos Usuários do SUS, principalmente para grupos de baixa renda. Muitos autores afirmam que os custos financeiros para incluir as PICs no sistema público de saúde se apresentam menor ou com mínima diferença significativas em relação ao uso da medicina convencional.

“Os números do SUS são, sempre, de grandes dimensões. Ao pensarmos que i5o milhões de pessoas são clientes do SUS, todo o custo que puder ser reduzido é significativo e pode trazer mudanças no fluxo de atendimento." (GUTIERREZ, 2008).

No curto prazo, considerando Mudanças organizacionais, preparação de profissionais, procedimentos e insumos utilizados, não se torna vantagem pois irá gerar grande custo, mas a médio e longo prazo, teria uma diminuição significativa nos custos e por conta da prevenção de doenças e promoção da saúde que essas terapias podem fornecer. Segundo o estudo de Lemonica (2014) sobre as diretrizes da Portaria Ministerial n. 971 voltadas a 
homeopatia, os profissionais da saúde se queixam da falta de incentivo financeiro do governo por conta de fortes resistências à produção científica homeopática.

No Brasil, terapias complementares raramente utilizadas no SUS podem estar relacionadas a falta de conhecimento de tais práticas por parte dos profissionais de saúde e, por sua vez, profissionais de saúde desconhece a existência da PNPIC, e não há matéria que trate do assunto na graduação. Porém, apesar dessa situação, alguns aspectos ainda são aceitáveis. Profissionais, acreditam na finalidade do seu tratamento e acreditam na Integração com a medicina tradicional.

\section{ATENÇÃO FARMACÊUTICA DE TRATAMENTO HOMEOPÁTICO PELO SUS}

Pacientes tratados com medicamentos homeopáticos devem cumprir um ciclo que inclui: o prescritor, o farmacêutico e pacientes, cada um cumpre seus deveres. O prescritor é responsável pela escolha correta de medicamentos usados por cada paciente; individualmente, e advertindo os usuários. Ao farmacêutico cabe a fabricação, a dispensação, a atenção e promoção ao uso racional dos medicamentos homeopáticos. Para o paciente é necessário atender às recomendações dos profissionais de saúde; pesquisar informações sobre sua condição clínica e medicamentos; e buscar em caso de dúvida ou efeitos adversos ajuda profissional.

Devido a homeopatia não ser uma medicina convencional para tratamento, não pode ser realizada sem medicamentos, então se entende logo que o farmacêutico deve estar presente no tratamento do paciente, desde a verificação da receita, até a dispensação correta e dar as orientações necessárias ao paciente.

\section{I PRINCIPAIS DOENÇAS TRATADAS COM A HOMEOPATIA}

\section{PROBLEMAS PSICOLÓGICOS}

A homeopatia auxilia no tratamento de transtornos psicológicos, como por exemplo a ansiedade, depressão, esquizofrenia, transtornos alimentares e estresse pós-traumático. Em geral, os sintomas do transtorno são abordados individualmente e é recomendada um acompanhamento psiquiátrico. 


\section{PROBLEMAS DE PESO}

Os medicamentos homeopáticos não trabalham de forma direta na perda de peso, mas nas disfunções que provocam o sobrepeso. Isso se dá porque a obesidade pode ser provocada por problemas relacionados à outras doenças como tireóide e deficiências metabólicas. A terapia floral também pode ser útil, já que os hábitos ruins podem ter relação com problemas emocionais.

\section{PROBLEMAS RESPIRATÓRIOS}

Rinite e sinusite por exemplo, podem te os efeitos reduzidos com a homeopatia. Alergias, asma e bronquite também são alvos comuns dos homeopatas. Porém boa parte dos tratamentos homeopáticos são de longo prazo e o paciente vai precisar esperar um certo tempo para que os resultados sejam positivos.

\section{CONCLUSÃO}

A proposta de inserção e identificação da homeopatia no SUS é um tema, digno de atenção. Além de tornar mais fácil para as pessoas obterem uma boa relação custobenefício, também é comprovada a eficácia, segurança e capacidade de personalizar o tratamento dos pacientes. Tendo em vista as pesquisas analisadas, a homeopatia pode atender a todos os requisitos e ter um lugar no sistema público de saúde. Os profissionais de saúde são importantes para mudar esse status da procura tão pequena pelo tratamento homeopático, pois esses são os mais responsáveis devido à falta de divulgação da importância da homeopatia para a sociedade.

Os profissionais farmacêuticos devem exercer a atenção sobre os medicamentos e buscar Atendimento humanizado e melhoria da qualidade de vida dos pacientes. A respeito disso Homeopatia e cuidados médicos trabalham juntos: em comparecer; buscar soluções mais ativas para os pacientes; fazer com que o paciente esteja no centro de cuidado da saúde.

Os princípios que orientam as políticas do SUS e que confirmam com os fundamentos da homeopatia é a humanização do atendimento aos pacientes, a adoção de práticas que 
previnem doenças. Além disso, a inclusão da homeopatia no SUS possibilita ampliar o universo de usuários, configurando o direito de escolha do cidadão.

"A homeopatia é uma terapêutica de ação generalista, que atua em todas as faixas etárias e requer tecnologia simples.” (NECKEL et al., 2010).

A implantação da homeopatia como uma opção nos serviços prestados pelo SUS pode trazer informações válidas e importantes para subsidiar a organização mais efetiva dessa terapêutica em diversos serviços. A integração da homeopatia no sistema de saúde publica pode reforçar os princípios de universalidade, integralidade e equidade.

\section{REFERÊNCIAS}

Souza, Gustavo. Leite, Ricardo. Yoshida, Edson. Santos, Nathalia. Uso Da Homeopatia No Sistema Único De Saúde. 2019. ıof. Revista Saúde Em Foco - Edição № ir. Curso De Graduação Em Farmácia. Faculdade Sudoeste Paulista (Fsp). Itapetininga, Sp. Disponível Em: Https://Portal.Unisepe.Com.Br/Unifia/Wp-

Content/Uploads/Sites/rooor/2019/03/o27_Uso-Da-Homeopatia-No-Sistema\%C3\%9anico-De-Sa\%C3\%9ade.Pdf Acessado Em: 15/03/2021.

Loch-Neckeli, Gecioni. Carmignanii, Françoise. Crepaldii, Maria. A Homeopatia No Sus $\mathrm{Na}$ Perspectiva De Estudantes Da Área Da Saúde. 2010. 7f. Rev. Bras. Educ. Med. Vol.34 No.Rio De Janeiro. Universidade Federal De Santa Catarina, Florianópolis, Sc, Brasil. Disponível Em: Https://Www.Scielo.Br/Scielo.Php?Pid=Soroo55022010000I000Io\&Script=Sci_Arttext Acessado Em: 15/03/2021.

Silva, Carola. Atenção Farmacêutica E Os Cuidados Na Administração De Medicamentos Homeopáticos. 2020. 2of. Trabalho De Conclusão De Curso (Artigo) Apresentado Ao Curso De Graduação Em Farmácia Da Universidade Federal Do Rio Grande Do Norte. Disponível Em: Https://Monografias.Ufrn.Br/Jspui/Bitstream/123456789/11187/r/Aten\%C $3 \% \mathrm{~A}_{7} \% \mathrm{C}_{3} \% \mathrm{~A}_{3}$ ofarmac\%C3\%Aauticaeoscuidados_Silva_2020 Acessado Em: 15/03/2021.

Souza, Alessandra. Implantação Do Tratamento Homeopático Na Rede Pública De Saúde. 2020. 7f. Revista Referências Em Saúde Da Faculdade Estácio De Sá De Goiás. Disponível Em: Http://Periodicos.Estacio.Br/Index.Php/Rrsfesgo/Article/Viewfile/8og6/4796667I Acessado Em: 15/03/2021. 
Monteiro, Dalva. Iriart, Jorge. Homeopatia No Sistema Único De Saúde: Representações Dos Usuários Sobre O Tratamento Homeopático. 2007. I8f. Cad. Saúde Pública Vol 23. Rio De Janeiro. Disponível Em: Https://Www.Scielo.Br/Scielo.Php?Script=Sci_Arttext\&Pid=Soro2-311x2007000800or7. Acessado Em: 20/05/2021.

Gutierrez, Marcia. Homeopatia No Sus. 2008. 8f. Pharmacia Brasileira Janeiro/Fevereiro. Disponível Em:

Https://Www.Cff.Org.Br/Sistemas/Geral/Revista/Pdf/5/ogar6_Homeopatia_No_Sus.P df. Acessado Em: 20/05/202I.

Simoni, Carmem. Benevides, Iracema. Política Nacional De Práticas Integrativas E Complementares No Sus - Pnpic Sus Trajetória De Avanços E Desafios. 2017. 3 f. Revista Aps, V.io, N.I. Disponível Em: Https://Www.Ufjf.Br/Nates/Files/2009/12/Pnacional.Pdf. Acessado Em 20/05/202I.

Neckel, Gecioni. Carmignan, Françoise. Crepaldi, Maria. A Homeopatia No Sus Na Perspectiva De Estudantes Da Área Da Saúde. 2010. 18f. Rev. Bras. Educ. Med. Vol.34 No.I. Rj. Disponível Em: Https://Www.Scielo.Br/Scielo.Php?Pid=Soroo55022010000100010\&Script=Sci_Arttext. Acessado Em: 20/05/2021. 